\title{
A case of biventricular endomyocardial fibrosis complicated by right ventricular outflow tract aneurysms
}

\author{
R Gonçalves, ${ }^{1} \mathrm{MB}$ ChB, MMed (Int Med), Cert Card (CMSA); R Meel, ${ }^{2}$ MB ChB, MMed (Int Med), Cert Card (CMSA) \\ ${ }^{1}$ Department of Cardiology, Charlotte Maxeke Johannesburg Academic Hospital and University of the Witwatersrand, Johannesburg, South Africa \\ ${ }^{2}$ Department of Cardiology, Chris Hani Baragwanath Academic Hospital and University of the Witwatersrand, Johannesburg, South Africa
}

Corresponding author: R Gonçalves (drrgoncalves@gmail.com)

Endomyocardial fibrosis remains a major public health problem worldwide. It is a restrictive cardiomyopathy, of uncertain aetiology, which may lead to right, left or biventricular heart failure. Progress continues to be made in understanding the prevalence and natural history of this disease. Specific treatment, apart from surgery, remains suboptimal. We report a case of advanced, biventricular EMF complicated by right ventricular outflow tract aneurysms.

S Afr Med J 2016;106(6):582-584. DOI:10.7196/SAMJ.2016.v106i6.9056

Endomyocardial fibrosis (EMF), despite being the most common restrictive cardiomyopathy worldwide, still remains poorly understood. ${ }^{[1,2]}$ Although first described in Uganda, ${ }^{[3]}$ this condition has been documented throughout the world, particularly in tropical and subtropical zones in Africa, Asia and South America. ${ }^{[1,2]}$ Its aetiology remains elusive. ${ }^{[1,2]}$ It possibly has different aetiologies in different parts of the world, with a similar final common pathway, although this remains conjecture. Hypotheses ranging from infectious and allergic causes to malnutrition and toxins have been postulated, although none has been rigorously tested. The condition is associated with fibrosis of the endocardium (extending into the myocardium) of the right and left ventricles, involving predominantly the apices and inflow regions, resulting in impaired filling of one or both ventricles. ${ }^{[1,2]}$ Patients may then present clinically with right, left or biventricular failure. Associated mitral and/or tricuspid regurgitation is common owing to frequent involvement of the papillary muscles with tethering to the ventricular wall.

Recently, a high prevalence of predominantly asymptomatic cases has been described in Mozambique, suggesting that the disease may be underdiagnosed in neighbouring South Africa (SA). ${ }^{[4]}$ We report a case of advanced biventricular EMF complicated by right ventricular outflow tract (RVOT) aneurysms, with the intention of stimulating clinicians to search for this condition.

\section{Case report}

A 49-year-old woman, originally from southern Mozambique, presented with an 8-year history of symptoms and signs of rightsided heart failure. She was initially misdiagnosed as having constrictive pericarditis. She originally presented with symptoms of exertional dyspnoea (grade II New York Heart Association (NYHA)), associated with peripheral oedema, that eventually progressed to anasarca. No history of rheumatic fever was reported. At the time of her assessment she was taking oral spironolactone $25 \mathrm{mg}$ daily, furosemide $80 \mathrm{mg}$ bid, potassium chloride $1200 \mathrm{mg}$ bid, atenolol $25 \mathrm{mg}$ daily, hydrochlorothiazide $25 \mathrm{mg}$ daily (added to address loop diuretic resistance) and warfarin according to her international normalised ratio (INR).
On examination, a raised jugular venous pressure was accompanied by anasarca (with prominent ascites). No jaundice, pallor or clubbing were noted. Pulse was irregular, and of variable volume consistent with atrial fibrillation (AF). The apex beat was undisplaced and normal in character. The heart sounds were normal and no signs of pulmonary hypertension were noted. Murmurs consistent with both tricuspid and mitral regurgitation were noted. The liver was impalpable due to the anasarca. The remainder of the examination was unremarkable. A urine dipstick revealed a trace of protein.

An electrocardiogram revealed $\mathrm{AF}$ with a ventricular rate of 84 beats/min, slurring of the terminal portion of the R-wave and a prominent R-wave in V2 (R:S ratio was 0.9). Her chest X-ray revealed an increased cardiothoracic ratio (0.7) with a globular heart shadow. This was due to left and right atrial dilation. A bulge was noted on the left heart border. No pleural effusions or pericardial calcification was present. Pulmonary venous congestion or pulmonary artery dilation was absent. Blood results indicated a normal full blood count; urea, creatinine and electrolytes; a therapeutic INR; and mildly deranged liver enzymes in keeping with hepatic congestion. She was HIV-negative.

Echocardiography revealed severely dilated atria (left atrial width $5.1 \mathrm{~cm}$ and right atrial width $5.3 \mathrm{~cm}$ ), mildly dilated left ventricle (LV) (LV internal diameter end diastole $5.52 \mathrm{~cm}, \mathrm{LV}$ internal diameter end systole $3.48 \mathrm{~cm}$ ) and a normal right ventricle (Fig. 1). LV wall thickness (basal) was within normal limits (interventricular septum end diastolic and LV posterior wall end diastolic diameters were $0.75 \mathrm{~cm}$ and $1.29 \mathrm{~cm}$, respectively), although the posterior wall was thicker than the septum. LV ejection fraction was preserved (65\%) but the lateral S'-velocity, on tissue Doppler imaging of the mitral annulus, was markedly diminished. Both ventricular apices were obliterated. No thrombi were noted in the atria or the apices. The E:E' ratios, at both the medial and lateral mitral annulus, were over 10, consistent with diastolic dysfunction. Severe tricuspid regurgitation and moderate mitral regurgitation were noted. The mechanism of regurgitation was due to tethering of the leaflets owing to papillary muscle shortening and annular dilation, as the chordae and leaflets were normal, the jets were central, and there was tip-totip coaptation of the leaflets of atrioventricular valves. The aortic 


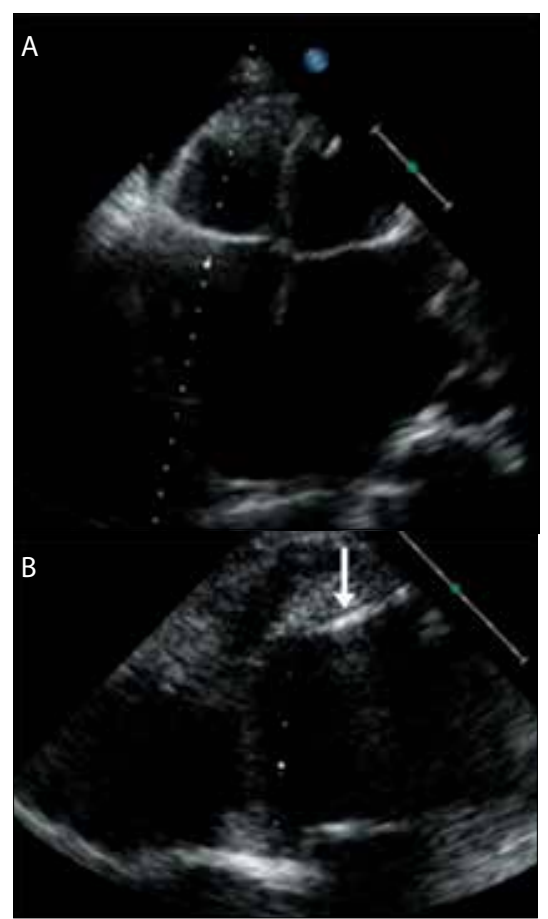

Fig. 1. (A) Echocardiogram (apical four chamber views) demonstrating massively dilated atria, small ventricles and obliterated right and left ventricular apices; (B) Calcification (arrow) in the left ventricular apex can be seen on the zoomed-in image.

and pulmonary valves were spared. The estimated systolic pulmonary artery pressure was $37 \mathrm{mmHg}$. No features consistent with pericardial effusion nor constriction were present. In summary, these findings were in keeping with a restrictive cardiomyopathy, most likely biventricular EMF.

Cardiac catheterisation was performed. This revealed a mean right atrial pressure (RAP) of $14 \mathrm{mmHg}$. Dynamic respiratory manoeuvers did not influence the RAP. The right ventricular (RV) systolic, diastolic and end-diastolic pressures (RVEDPs) were 33, 4 and $15 \mathrm{mmHg}$, respectively. The systolic, diastolic and mean pulmonary artery pressures were 37,14 and $23 \mathrm{mmHg}$, respectively (normal). Mean pulmonary capillary wedge pressure was $20 \mathrm{mmHg}$ and pulmonary arteriolar resistance was 58 dynes.s $/ \mathrm{cm}^{5}$ (normal). LV systolic, diastolic and enddiastolic pressures (LVEDPs) were 110, 4 and $14 \mathrm{mmHg}$, respectively. A dip and plateau sign was seen in both ventricles and LVEDP to RVEDP pressure difference of $1 \mathrm{mmHg}$ was recorded. Ventricular interdependence was absent during deep respiration on simultaneous left and right ventricular pressure measurements. Obliteration of both ventricular apices and distortion of the normal ventricular outline was noted on right and left ventriculography (Figs 2 and 3). An inferior

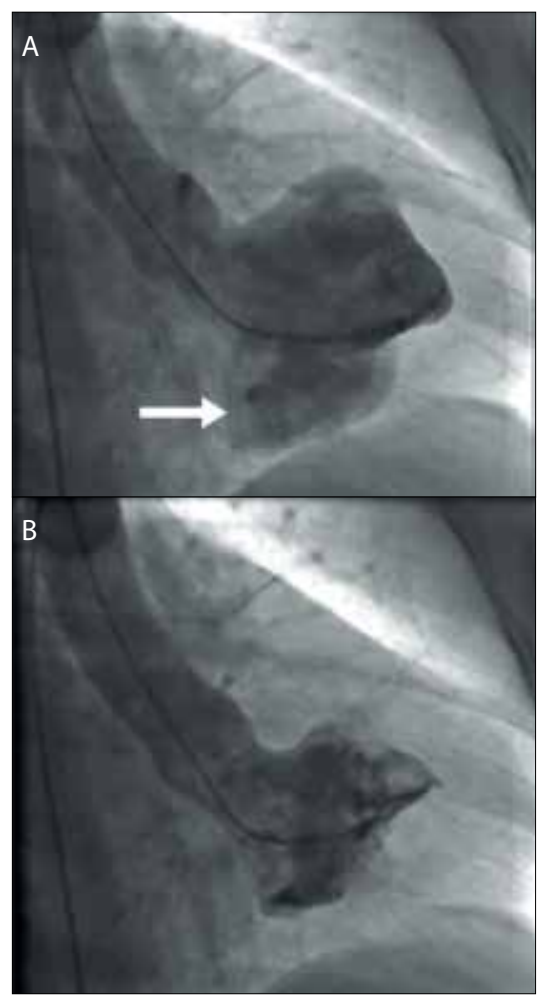

Fig. 2. A left ventriculogram showing distortion of the left ventricular cavity with an inferior wall aneurysm (arrow) and obliteration of the apex. (A) Diastole; (B) Systole.

wall LV aneurysm was apparent in diastole. Two tubular aneurysms originating from the RVOT were noted, with dilation of the RVOT. These structures did not communicate with another chamber and emptied during systole. Pulmonary artery saturation was $76 \%$ and aortic saturation was $96 \%$. The cardiac output was estimated at $4.1 \mathrm{~L} / \mathrm{min}$ (cardiac index $2.65 \mathrm{~L} / \mathrm{min}$ ). The coronary arteries were normal. An endomyocardial biopsy was not performed.

These findings were corroborated by cardiac magnetic resonance imaging (MRI) (Fig. 4). Markedly dilated atria and thickening of the ventricular endocardium, with obliteration of both apices, were noted. Papillary muscle thickening and shortening probably contributed to the tricuspid and mitral regurgitation. No thrombi were noted.

After a discussion with the patient, surgery was deferred, given the high surgical risk associated with the procedure. She was continued on the afore-mentioned medical therapy.

\section{Discussion}

This case report highlights a unique example of biventricular EMF complicated by RVOT aneurysms. The classically described pathological changes in right-sided EMF include obliteration of the right ventricular

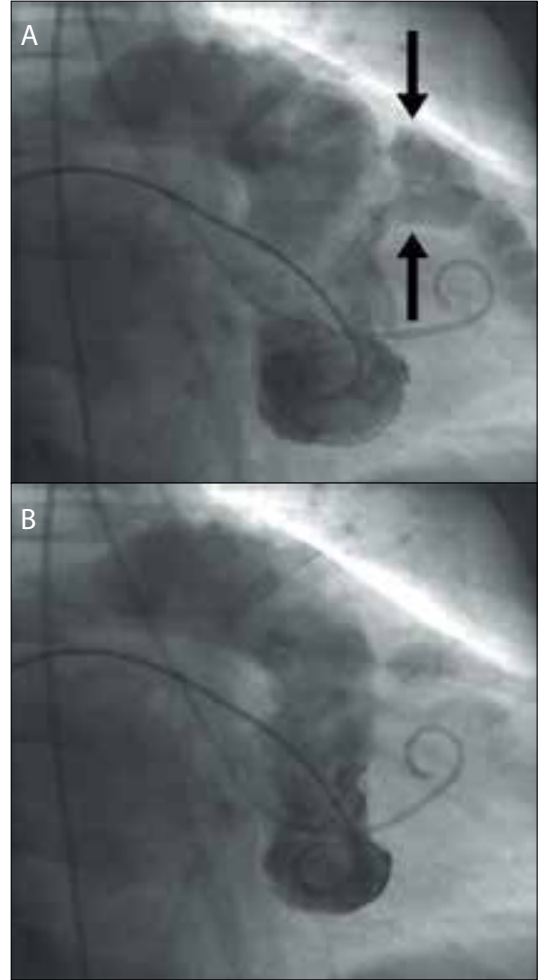

Fig. 3. A right ventriculogram demonstrating distortion of the right ventricular cavity with obliteration of the apex, dilation of the RVOT and two tubular aneurysms (arrows) originating from the RVOT (both of which empty during systole). (A) Diastole; (B) Systole.

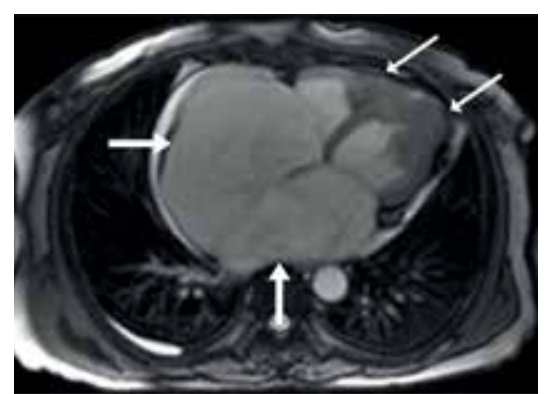

Fig. 4. Cardiac MRI showing bilaterally dilated atria (thick arrows), small ventricles and obliteration of the ventricular apices (thin arrows). Papillary muscle thickening and shortening, a normal pericardium, and the absence of thrombi are noted.

apex by fibrosis, dilation of the RVOT, tricuspid regurgitation and a massively dilated right atrium. ${ }^{[5-8]}$ In left-sided disease, partial LV obliteration by fibrosis occurs. Further extension of the process into the inflow tract causes papillary muscle adhesion to the LV wall leading to subsequent mitral regurgitation. The left atrium is dilated, although frequently less so than the right atrium.

Interestingly, early radiological studies occasionally identified a convex bulge, high on the LV border on the chest X-ray, 
which was due to diastolic distention of the RVOT based on flouroscopy ${ }^{[5,6]}$ This feature was present in this case. The presence of focal, tubular aneurysms of the RVOT, however, is unique. These may have arisen owing to high RV diastolic pressures related to reduced RV volume, decreased RV compliance and poor RV relaxation. The RVOT is the most likely region to dilate, given its relatively thin wall and the absence of fibrosis in this region. A similar explanation may account for the LV inferior wall aneurysm that was observed in this case.

A recent study performed in Mozambique has contributed significantly to the field. ${ }^{[4]}$ It not only highlighted the prevalence of this disease in that country but also defined a set of echocardiographic criteria that are useful both for diagnosis and assessment of severity. ${ }^{[4]}$ The estimated overall prevalence of EMF was $19.8 \%$, with the highest prevalence among persons 10 - 19 years of age (28.1\%), more commonly in males. Biventricular EMF was most common (55.5\%), followed by right-sided EMF (28\%), and the minority, (16.5\%) were left sided. The majority of patients were asymptomatic (77.3\%), with most cases diagnosed only on echocardiography. Echocardiography is an essential tool for the diagnosis of EMF.

The proposed echocardiographic criteria for diagnosis and assessment of the severity of EMF are summarised briefly. ${ }^{[4]} \mathrm{A}$ definite diagnosis of EMF was made in the presence of 2 major or 1 major and 2 minor criteria. The severity was classified as a total score, with $<8$ indicating mild disease, 8 - 15 moderate disease and $>15$ severe disease (the maximum possible score being 35 points). The major and minor criteria follow with the point scores indicated in parentheses. The major criteria were as follows: endomyocardial plaques $>2 \mathrm{~mm}$ in thickness $(2)$; thin $(\leq 1 \mathrm{~mm})$ endomyocardial patches affecting more than one ventricular wall (3); obliteration of the RV or LV apex (4); thrombi or spontaneous contrast without severe ventricular dysfunction (4); retraction of the RV apex (RV apical notch) (4); and atrioventricular valve dysfunction due to adhesion of the valvular apparatus to the ventricular wall $(1-4$, depending on the severity of regurgitation). The minor criteria were as follows: thin endomyocardial patches localised to one ventricular wall (1); restrictive flow pattern across mitral or tricuspid valves
(2); pulmonary valve diastolic opening (2); diffuse thickening of the anterior mitral leaflet (1); enlarged atrium with normal-size ventricle (2); m-mode movement of the interventricular septum and flat posterior wall (1); and enhanced density of the moderator or other intraventricular bands (1). Based on this scoring system, our patient had a score of least 14 , indicating moderate disease.

The differential diagnosis includes dilated cardiomyopathy, constrictive pericarditis, tuberculous pericarditis and rheumatic heart disease.$^{[1,2]}$ These conditions have a high prevalence in the areas where EMF is endemic, although a careful clinical and echocardiographic review will usually yield the diagnosis. Ebstein's anomaly may mimic right-sided EMF. Furthermore, other restrictive cardiomyopathies such as amyloidosis, haemochromatosis and sarcoidosis may pose a diagnostic dilemma. Cardiac MRI would be useful in this regard if the diagnosis remains in doubt. Cardiac malignancies constitute another possible diagnostic group. The treatment has been reviewed elsewhere in detail and will not be discussed. ${ }^{[1]}$

\section{Conclusion}

This case report highlights the importance of considering the diagnosis of EMF, in particular in patients originally from endemic areas. Although EMF is not endemic in SA, SA has a large migrant population from countries where the disease is endemic, in particular Mozambique. For this reason, persistent vigilance is necessary to diagnose and correctly manage this fascinating disease.

1. Mocumbi A, Yacoub S, Yacoub M. Neglected tropical cardiomyopathies: II. Endomyocardial fibrosis Myocardial disease. Heart 2008;94(3):384-390. DOI:10.1136/hrt.2007.136101

2. Bukhman G, Ziegler J, Parry E. Endomyocardial fibrosis: Still a mystery after 60 years. PLoS Negl Trop Dis 2008;2(2):e97. DOI:10.1371/journal.pntd.0000097

Dis 2008;2(2):e97. DOI:10.1371/journal.pntd.0000097
Davies J. Endomyocardial fibrosis in Uganda. East Afr Med J 1948;25:10-16.

4. Mocumbi A, Ferreira M, Sidi D, Yacoub M. A population study of endomyocardial fibrosis in a rural 4. Mocumbi A, Ferreira M, Sidi D, Yacoub M. A population study of endomyocardial fib
area of Mozambique. N Engl J Med 2008;359(1):43-49. DOI:10.1056/NEJMoa0708629 area of Mozambique. N Engl J Med 2008;359(1):43-49. DOI:10.1056/NEJMoa0708629
Cockshott W, Sarić S, Ikeme A. Radiological findings in endomyocardial fibrosis. Circ 1967;35(5):913Cockshott W, Sarić S, Ikeme A. Radiological findings in endomyocardial fibrosis. Circ 1967,35t
922. DOI:10.1161/01.CIR.35.5.913
Cockshott W. Angiocardiography of endomyocardial fibrosis. Br J Radiol 1965;38:192-200.

6. Cockshott W. Angiocardiography of endomyocardial fibrosis. Br J Radiol 1965;38:192-200.
7. Connor D, Somers K, Hutt M, Manion W, D’Arbela P. Endomyocardial fibrosis in Uganda (Davies disease). Part I. An epidemiologic, clinical, and pathologic study. Am Heart J 1967;74:687-709.

8. Connor D, Somers K, Hutt M, Manion W, D'Arbela P. Endomyocardial fibrosis in Uganda (Davies disease). Part II. An epidemiological, clinical, and pathological study. Am Heart J 1968;74:107-124.

Accepted 7 March 2016. 